\title{
Influence of electro-acoustic coating of metals on ordering of dislocating structures
}

\author{
Andrei Kochetov ${ }^{1}$, Elena Fisunova ${ }^{1}$, Tatyana Lavrenova ${ }^{1,{ }^{*}}$ and Olga Baryshnikova ${ }^{1}$ \\ ${ }^{1}$ FGBOU VO «Don State Technical University» (DSTU), 344000, Rostov-on-Don, Russian \\ Federation
}

\begin{abstract}
Method of electroacoustic spraying allows getting ordering of material remote structures by complex energy interaction and ultrasound field. Complex interaction influences on strengthening surface is leading to a thin film formation, which gives a strong base for the basic material by formation of forecasting structural phase contain for getting protective wear-resistant films and also thanks to interaction forces between a film and a particle location.
\end{abstract}

\section{Introduction}

The increasing problem of life service for machine components and formation instrument is the burning problem also for local and foreign producers of manufacturing production. This problem solving is closely connected with modern strengthening technology usage, based on using of highly-concentrated energy sources which allow to get ordering of material remote structures.

Obtaining ordered dislocation structures, under the influence of a powerful longitudinaltorsional ultrasonic field and an electric spark in metals, can stimulate the development of a number of scientific and applied works in this field. The method electroacoustic coating is newest wear resistance technology. Basically of this method was put interaction of high concentration sources energy a lot of electric power and ultrasonic field. This method allows obtaining wear resistance coating thickness from 2 to $5 \mathrm{mcm}$. Process of fixing of covering is ecological clean.

\section{Simplified analytical model of interaction of a single dislocation with the obtained layers by electroacoustic sputtering}

Since 1934, when Rosky [1-5] established that an oxide film with a thickness of less than 20 atomic layers increases the critical shear stress of cadmium single crystals by more than $50 \%$, the question of the effect of solid surface films on the behavior of crystals under load is constantly in the field of view of scientists.

\footnotetext{
* Corresponding author: bys ka87@mail.ru
} 
Currently in many cases researchers have achieved much more success in the development of acceptable hardening technologies than in the physical justification of the processes themselves, as well as methods for predicting hardening coatings and their properties, depending on the conditions of subsequent operation.

This situation often leads to unjustified ignoring and sometimes misunderstanding of the need for depth physical studies of micro-processes of hardening and related phenomena from the standpoint of fundamental Sciences. On the other hand, the advance in the development of applied issues is due not only to obvious utilitarian and pragmatic considerations, but also to the fact that to some extent this is due to the objective difficulties of using common physical approaches and model representations in relation to the phenomena accompanying the processes of hardening, friction and wear. The mathematical apparatus is poorly developed, i.e. researchers are forced to solve the problem of hardening at the engineering level.

The absence of reliable mathematical models of hardening processes that do not allow to take into account the stochastic (random) nature of many related phenomena, does not allow to objectively assess the effectiveness of various hardening technologies.

The double barrier created by electroacoustic sputtering prevents dislocations from reaching the surface. The conclusion about the barrier mechanism of influence is confirmed by numerous experiments conducted with the use of various crystals-bases and applied surface layers (both oxide [1, 2] and metal [3, 6]) confirmed in most cases the effect found by Rosky. On the basis of the stated material the simplified analytical model of interaction of a single dislocation with the received layers by a method of electroacoustic sputtering is offered (Fig. 1).

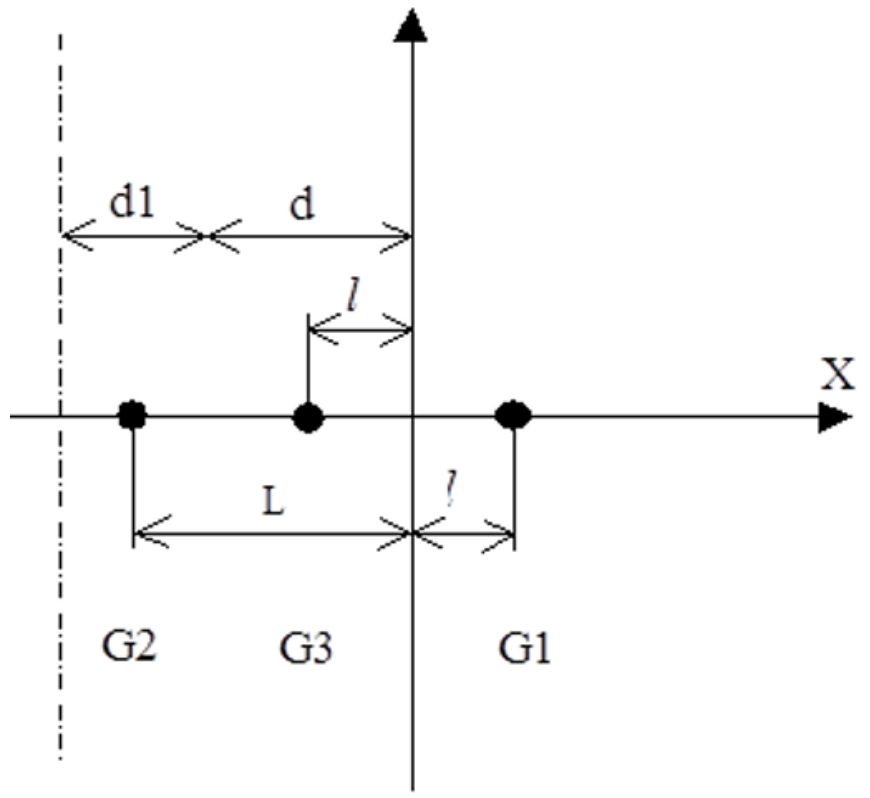

Fig. 1. A single dislocation near the interface "coated layer-metal": $G_{1}$ - the shear modulus of the material of the hardened sample; $G_{2}$ - is the shear modulus of the deposited layer; $G_{3}$ - is the shear modulus of plastically deformed layer; $d$ - is the thickness of plastically deformed layer; $\mathrm{d} 1$ is the thickness of the sprayed layer; $l$ - the distance from the dislocation to the boundary of section; $L$ - is the distance from the location of the image of the deposited layer to the interface. 
Let us now analyze the case when a compound with a shear modulus is formed at the film - metal boundary. If we limit ourselves to the case of a "thick layer", the force acting on the dislocation in the metal from the side of the intermediate layer of thickness, having a shear modulus, is equal to:

$$
F_{1}=\frac{G_{1} \cdot b^{2} \cdot K_{31}}{4 l \pi} \cdot\left\{1-\left(1-K_{31}^{2}\right) \cdot \sum_{n=1}^{\infty}\left(-K_{23}\right)^{n} \cdot K_{31}^{n+2} \cdot \frac{\alpha}{\alpha+n}\right\},
$$

where $K_{i j}=\left(G_{i}-G_{j}\right) /\left(G_{i}+G_{j}\right)$.

To determine the force of interaction of the dislocation with the film, we find the total sum of forces acting on the real dislocation from an infinite number of dislocations «image»:

$$
F_{1}=\frac{G_{1} K b^{2}}{4 l \pi} \cdot\left\{1-\left(1-K^{2}\right) \cdot \sum_{n=1}^{\infty} K^{n-1} \frac{\alpha}{\alpha+n}\right\}
$$

where $\alpha=l / d$.

Depending on the ratio of the shear modulus, the "image" force can have a different character [6].

Using the principle of superposition, we can say that the resulting force acting on the part of the obtained layers on the dislocation in the metal is determined as the sum of the forces applied to it

$$
F_{p}=F_{1}+k F_{2}
$$

where $k \approx l / L$.

\section{Summary}

On the basis of theoretical and experimental studies of the effect of surface films on the ordering of dislocation structures obtained by electroacoustic sputtering, the following conclusions can be made:

The most acceptable explanation for crystal hardening is surface films, which prevent dislocations from reaching the surface by suppressing slip and ordering dislocation structures.

If the shear modulus of the crystal-base is less than the shear modulus of the deposited layer, the force acting on the part of this layer tends to push the dislocation away from the phase boundary, which increases the strength of the product.

The higher the film modulus (i.e., the greater), the smaller the number of dislocations in the cluster at the same applied voltage. Thus, a crystal covered with a rigid film will plastically deform less than a crystal without a film under the same stress (provided that the average path length of dislocations in the crystal is not very small). This may be the mechanism of primary hardening of crystals with rigid coatings at an early stage of deformation. 


\section{References}

1. E.N. da C. Andrade, C. Henderson, Phil. Trans., 244A, 177, (1981).

2. J.J. Gilman, Trans. Amer. Inst. Min. a. Metall. Engrs., 191, 1148, (1981).

3. M. P. Piskus, E.R. Parker. Trans. Amer. Inst. Min. a. Metall. Engrs., 191, 792, (1990).

4. J.J. Gilman, Amer. Soc. Test. Mater., Spes. Techn. Publ. №171, 3, (1985).

5. A.K. Head, Austr. J. Phys., 13, 278, (1985).

6. F. R. Lipsett, R. King, Proc. Pris. Soc. Lond., 70B, 608, (1997). 